\title{
Erratum to: Ubiquitin-conjugating enzyme complex Uev1A-Ubc13 promotes breast cancer metastasis through nuclear factor-kB mediated matrix metalloproteinase-1 gene regulation
}

Zhaojia Wu ${ }^{1,2}$, Siqi Shen ${ }^{1}$, Zhiling Zhang ${ }^{1}$, Weiwei Zhang ${ }^{1}$ and Wei Xiao ${ }^{1,2^{*}}$

\section{Erratum}

After the publication of this work [1] an error was noticed in Fig. 2d, in which an image from UEV1C transfected cells without Dox treatment (Dox ${ }^{-}$) was mistakenly presented as MMS2 transfected Dox- cell image. The corrected Fig. 2 that contains a replacement image for MMS2 transfected Dox cells in Fig. 2d is presented. As UEV1C and MMS2 transfections have indistinguishable effects on cell invasion (Fig. 2e), the correction does not affect our conclusions. Nevertheless, we apologize for this error.

Received: 6 March 2017 Accepted: 6 March 2017

Published online: 28 March 2017

\section{Reference}

1. Wu Z, Shen S, Zhang Z, Zhang W, Xiao W. Ubiquitin-conjugating enzyme complex Uev1A-Ubc13 promotes breast cancer metastasis through nuclear factor-small ka, CyrillicB mediated matrix metalloproteinase-1 gene regulation. Breast Cancer Res. 2014;16:R75

\footnotetext{
*Correspondence: wei.xiao@usask.ca

${ }^{1}$ College of Life Sciences, Capital Normal University, Beijing 100048, China

${ }^{2}$ Department of Microbiology and Immunology, University of Saskatchewan, Saskatoon S7N 5E5, Canada
} 


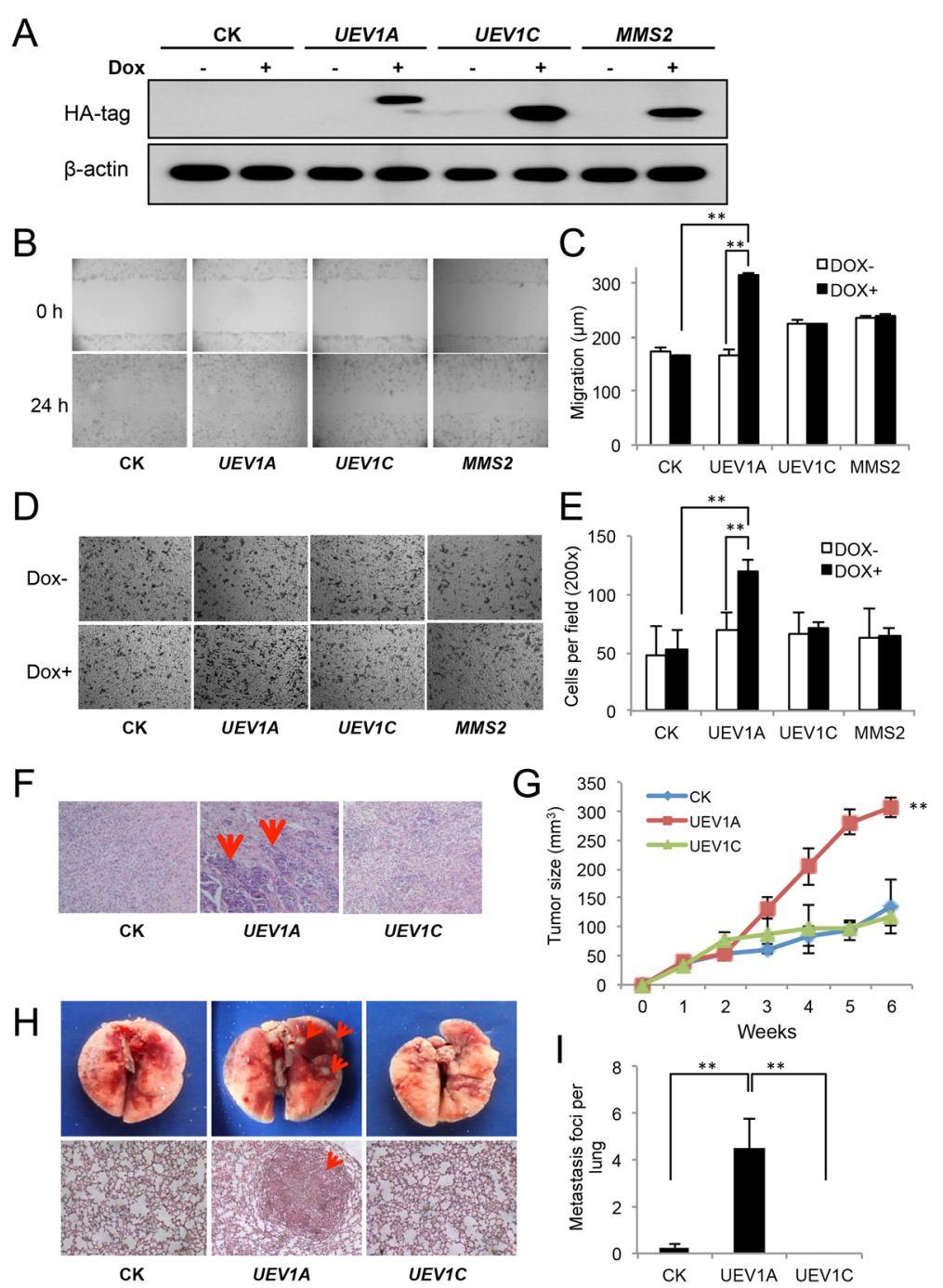

Fig. 2 UEV $1 A$ overexpression promotes breast cancer cell invasion in vitro and metastasis in a xenograft model. a pcDNA4.0/TO/HA(+) vector $(C K)$ expressing UEV1A, UEV1C or MMS2 was stably transfected into MDA-MB-231-TR cells, with or without doxycycline (Dox) treatment. The level of ectopic gene expression was monitored by western blot against an anti-HA antibody. $\mathbf{b}$ Representative images of wound-healing assays with Dox treatment. c Statistical analysis of cell migration of wound-healing assay with and without Dox treatment. The migration distance of cells was measured in five different wells in each group under a light-microscope. $\mathbf{d}$ Representative images of cell invasion assay with Matrigel-coated transwells. e Statistical analysis of the cell invasion assay data. Cells that invaded the lower surface of the filter were counted in five random fields under a light-microscope at 200x magnification. $\mathbf{f}-\mathbf{i}$ In vivo tumorigenesis and metastasis assays using a xenograft mouse model. $\mathbf{f}$ Lymph node sections after sacrifice were stained with H\&E. The lymph node metastasis sites are shown by red arrows. $\mathbf{g}$ Quantitative analysis of tumor growth. Tumor growth was measured every week after injection (Day 0 ) and expressed as mean \pm SD $(n=10)$. $\mathbf{h}$ The in vivo metastasis assay in xenograft mice. Upper panel, the lung metastasis nodules formed are shown by red arrows. Lower panel, the lung sections were stained with H\&E and the lung metastasis under a light-microscope at 100x magnification is indicated by a red arrow. $\mathbf{i}$ Quantitative analysis of the in vivo lung metastasis as measured by the number of metastasis foci per lung for all four sections ( $n=10$ mice for each treatment) 